\title{
ACL reconstruction with physiological graft tension by intraoperative adjustment of the anteroposterior translation to the uninjured contralateral knee
}

\author{
Johannes Dominik Bastian • Salvatore Tomagra • \\ Andreas J. Schuster • Stefan Werlen • \\ Roland P. Jakob • Matthias A. Zumstein
}

Received: 12 November 2012 / Accepted: 25 February 2013/Published online: 8 March 2013

(c) Springer-Verlag Berlin Heidelberg 2013

\begin{abstract}
Purpose Fixation of anterior cruciate ligament (ACL) substitutes with non-physiological anteroposterior translation (APT) worsens outcome. The aim was to present a technique for physiological APT adjustment of the transplant in ACL reconstruction and its outcome at midterm. Methods In a consecutive series of 28 patients (age $32 \pm 11$ years, 24 male), chronic ACL deficiency was treated by bone-patella-tendon-bone reconstruction. Transplant APT was adjusted to that of the contralateral uninjured ACL, measured 3, 6, and 12 months postoperatively using the Rolimeter ${ }^{\circledR}$. At a median follow-up of 5.3 years (3-8years), $82 \%$ of the patients were re-evaluated with APT measurement and using IKDC-, Tegner-, Lysholm-Scores, conventional radiographs and MRI.

Results No differences in APT (mean \pm SD) between uninjured and reconstructed knees were observed after adjustment ( $6 \pm 1$ versus $6 \pm 1 \mathrm{~mm}$, n.s.). Three months postoperatively, a statistically significant increase in APT $(7 \pm 1 \mathrm{~mm})$ and a further increase at midterm $(9 \pm 2 \mathrm{~mm})$
\end{abstract}

J. D. Bastian $(\bowtie) \cdot S$. Tomagra · M. A. Zumstein Department of Orthopedic and Trauma Surgery,

University of Bern, Inselspital, Freiburgstrasse 3,

3010 Bern, Switzerland

e-mail: johannesbastian@gmx.de

\author{
A. J. Schuster \\ Department of Orthopedic Surgery, Spital Netz Bern-Ziegler, \\ Bern, Switzerland
}

S. Werlen

Department of Radiology, Sonnenhof, Bern, Switzerland

R. P. Jakob

Department of Orthopaedic Surgery, Spital Tafers,

Tafers, Switzerland were observed. Patients scored "normal" or "nearly normal", respectively, in $79 \%$ (IKDC) and 4 (3-9) points (Tegner; median, range) or $89 \pm 9$ points (Lysholm; mean $\pm \mathrm{SD}$ ). Radiological evaluation showed no, minimal or moderate joint degeneration in 5, 20 and $75 \%$ of patients, respectively. MRI confirmed intact ACL transplants in all patients.

Conclusion ACL reconstruction using the presented technique was considered successful, as patients did not suffer from subjective instability, radiographic analysis did not provide evidence for graft rupture at midterm. However, APT increase and occurrence of degenerative changes in reconstructed knees at the midterm might not be prevented even by restoration of a physiological APT in ACL reconstruction. The Rolimeter can be used for quick and easy intraoperative indirect control of the applied tension to the ACL transplant by measuring the APT to obtain physiological tensioning resulting in a satisfying outcome at midterm.

Level of evidence IV.

Keywords Anterior cruciate ligament - Patella tendon . Anteroposterior translation · Outcome - Rolimeter ·

Reconstruction

\section{Introduction}

A reported sequela of anterior cruciate ligament (ACL) rupture is the occurrence of symptomatic osteoarthritis years after injury [21]. Surgical treatment of ACL deficiency provides ligamentous stability but did not prevent from occurrence of osteoarthritis [16]. The intraoperative fixation of the ACL substitute with non-physiological anteroposterior tension which predisposes patients to 
osteoarthritis $[10,22]$ may account for this observation. In addition, the amount and the application of the appropriate initial graft tension at the time of fixation are discussed controversially $[1,19]$. Surgeons apply graft tensions within the range of 20-80 N [3], whereas others accomplish graft tensioning "by hand" $[2,20]$. Thus, the restoration of a physiological knee laxity may be essential in ACL reconstruction, and a device is required to enable the surgeon to apply a physiological tension to the ACL substitute.

In the presented report, we defined "physiological knee laxity" in ACL-deficient knees as the individual anteroposterior translation (APT) of each patient's contralateral uninjured knee. To enable the surgeon to accomplish physiological knee laxity in reconstructed knees, the APT in affected knees was intraoperatively adjusted to that of the contralateral uninjured knees using a new mechanical testing device. It was hypothesized that restoration of physiological laxity using the device (1) is feasible and prevents from (2) recurrent joint instability as well as (3) radiological joint deterioration at midterm.

\section{Materials and methods}

Between 2002 and 2006, 59 patients (45 male; median age 33 year, range 16-58 years) with chronic ACL deficiency were treated by ACL reconstruction using autologous ipsilateral bone-patella-tendon-bone (BPTB) graft. The APT of the affected ("preOP") and uninjured contralateral knees ("uninjured") of each patient undergoing ACL reconstruction was preoperatively measured using a Rolimeter ${ }^{\circledR}$ (Don Joy Global Company; Vista, CAUSA) mechanical testing device as previously described [27]. Intraoperatively, the APT of the affected knee was adjusted to that of the contralateral knee in $20^{\circ}$ knee flexion.

Of 59 patients, only patients with (1) preoperative MRI scans, (2) uninjured contralateral knees, or without (3) previous surgery in the injured knee, (4) joint hypermobility according to the Beighton criteria [4] were included in the study protocol. In cases of injuries during the postoperative follow-up interval to the operated or contralateral knees, patients were intended to be excluded from the study. The study was approved by the local ethical committee (Kantonale Ethikkommission, KEK 085/09, Universität Bern). Informed consent to participate was provided by the patients.

\section{Surgical Technique}

ACL reconstruction was performed with the BPTB-technique [7, 18, 25]. Implantation consisted of an arthroscopically assisted, fluoroscopically controlled [29] ACL reconstruction technique. One bone block was wedge shaped and fixed in the femoral tunnel using an "aperture"pressfit blocking unitunnel technique with two incisions. Tibial bone block fixation occurred over a 3.5-mm cortical screw with a $1.5-\mathrm{mm}$ standard wire. To balance the initial APT to that of the uninjured contralateral knee, APT was measured intraoperatively on both knees with the Rolimeter $^{\circledR}$ in triplicate before graft fixation as described previously [27]. All surgeries were performed by the same, board-certified knee surgeon (A. J. S.).

Clinical evaluation

The APT was measured at each time point using the Rolimeter $^{\circledR}$ with additional measurement using the KT-1000 arthrometer [11, 27] at the midterm. Because side-to-side difference $\geq 3 \mathrm{~mm}$ has been previously considered indicative of ACL deficiency [11], side-to-side difference $\geq 3 \mathrm{~mm}$ was defined as graft failure in our series. At midterm, the subjective and clinical assessment included the International Knee Documentation Committee (IKDC) Knee Ligament Evaluation Form [15, 17], Tegner Activity Level [30], Lysholm Knee Scoring Scale [30] and NoyesScore 24]. The need for revision surgery and incidence of graft rupture during follow-up were also assessed. These assessments were performed by an independent observer to the original procedure (S. T.).

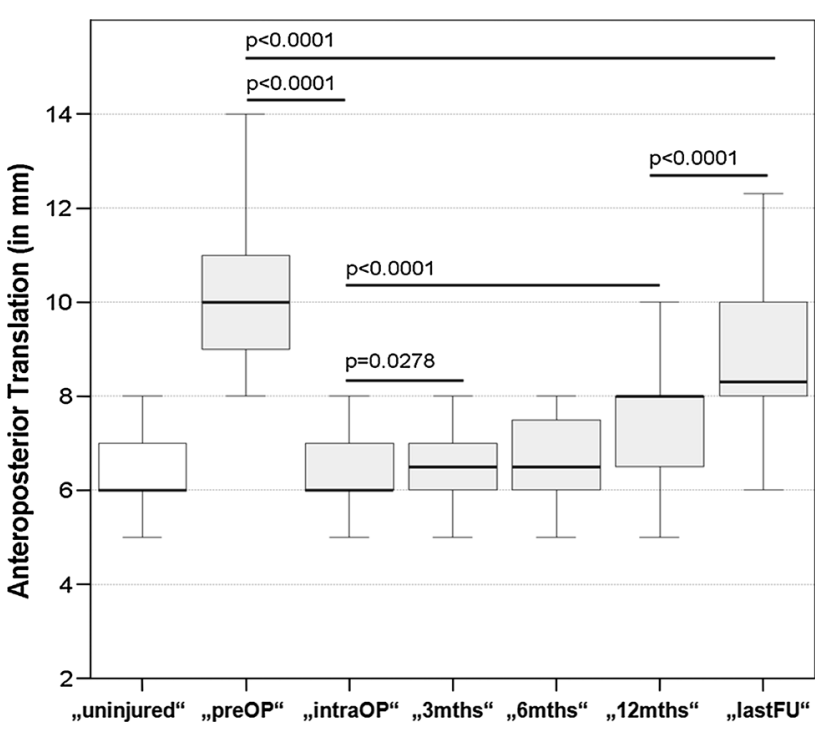

Fig. 1 Box and whisker plots show the values of Rolimeter APT measurements for uninjured contralateral knees ("uninjured", white box) and for affected knees (grey boxes) preoperatively ("preOP"), intraoperatively ("intraOP"), at 3,6 and 12 months postoperatively ("3 months", "6 months", "12 months", respectively), and at the last follow-up ("lastFU"). The horizontal line indicates the median value, the top and bottom borders of the box show the 25th and 75th percentiles, whiskers show the 10th and 90th percentiles. $P$ values for comparisons with statistical significance are shown 
Evaluation of conventional radiographs and MR imaging

Bipedal posteroanterior radiographs in weight-bearing at $30^{\circ}$ flexion, monopodal lateral radiographs in weightbearing at $20^{\circ}$ flexion and patellofemoral radiographs in supine position at $45^{\circ}$ flexion were obtained from the uninjured contralateral and operated knee at midterm. The radiological outcome was assessed according to IKDC recommendations [15].

To radiographically assess APT at midterm, the medial anterior tibial translation of affected and contralateral uninjured knees was measured in a monopodal stance test as previously published [6]. The difference in medial anterior tibial translation in the monopodal stance test between knees of each patient was calculated. The frequencies indicative for ACL deficiency obtained from both measurements (either clinically in supine position using the Rolimeter $^{\circledR}$ or radiographically under full weight-bearing conditions with isometric muscle activation in the monopodal stance test) were compared to each other to detect potential effects of perigenicular musculature activation in stabilizing potentially ACL-deficient knees.

Magnetic resonance imaging studies were performed preoperatively to confirm the clinical diagnosis of torn ACL [26]. Additionally, MR imaging was performed at the last follow-up to assess the integrity of the ACL reconstruct on a Trio 3 Tesla unit (Siemens, Erlangen, Germany). Sagittal isotropic pd-space sequences with and without fat saturation (thickness $0.6 \mathrm{~mm}$ ) and coronal TIRM sequences (thickness $3 \mathrm{~mm}$ ) were used; scan time was $20 \mathrm{~min}$. Radiographic evaluation was performed by an independent, experienced radiologist (S. W.).

\section{Statistical analysis}

Differences in the measured APT at different time points were analyzed with a linear mixed-effects model including fixed time effects and random subject effects. All pairwise comparisons between two time points were performed, and $P$ values were adjusted by the Tukey-Kramer method. The differences in measured APT between the reconstructed knee and contralateral uninjured knee of each patient were analyzed by $\mathrm{t}$ tests for paired data. Since the main interest was to check equivalence between the APT of uninjured and reconstructed knees, $90 \%$ confidence intervals for the difference were calculated to determine whether they fell within a prespecified range of $\pm 1 \mathrm{~mm}$. For comparisons of APT measurements between the Rolimeter ${ }^{\circledR}$ and the monopodal stance test, the Wilcoxon signed-rank test was used; for comparison of binominal data (APT side-to-side difference $\geq 3 \mathrm{~mm}$ ), the McNemar test was used. Calculations were performed with SAS 9.2 (SAS Institute Inc.,
Table 1 IKDC Grading (in \%)

\begin{tabular}{|c|c|c|c|c|}
\hline IKDC & $\begin{array}{l}\text { Normal } \\
\text { (Grade } \\
\text { A) }\end{array}$ & $\begin{array}{l}\text { Nearly } \\
\text { normal } \\
\text { (Grade } \\
\text { B) }\end{array}$ & $\begin{array}{l}\text { Abnormal } \\
\text { (Grade C) }\end{array}$ & $\begin{array}{l}\text { Severely } \\
\text { abnormal } \\
\text { (Grade } \\
\text { D) }\end{array}$ \\
\hline $\begin{array}{l}\text { Patient subjective } \\
\text { assessment }\end{array}$ & 52 & 39 & 9 & - \\
\hline Symptoms & 52 & 39 & 9 & - \\
\hline Range of motion & 96 & - & 4 & - \\
\hline Ligament examination & 70 & 30 & - & - \\
\hline Harvest site morbidity & 78 & 22 & - & - \\
\hline Overall evaluation & 22 & 57 & 22 & - \\
\hline
\end{tabular}

Cary, NC, USA). For further demonstration of clinical and radiographic results, $P$ values are not provided because the statistical analysis used was descriptive.

\section{Results}

In all patients, surgery was finished with no intraoperative complications. No postoperative complications occurred, and no revision surgeries were needed. Twenty-eight patients matched the inclusion criteria and were monitored in a prospective surgical evaluation protocol with examinations 3 ("3 months"), 6 ("6 months") and 12 ("12 months") months postoperatively. For re-evaluation at a median follow-up of 5.3 years (range: $3-8$ years), 23 patients (19 male; median age 31 years, range $18-58$ years) were available. No patient had to be excluded due to injuries to the operated or contralateral knee. Two of 28 patients refused consultation because they were doing well. Three patients were lost to follow-up (2 moved overseas, and one could not be traced).

The preoperative measured APT of the affected knee, $10.5 \pm 1.7 \mathrm{~mm}$, was significantly reduced to $6.0 \pm 0.8$ $\mathrm{mm}(P=0.0001)$, equivalent to the APT of the corresponding contralateral uninjured knees $(6.3 \pm 0.8 \mathrm{~mm}$; n. s.). The measured APT in reconstructed knees increased to $6.9 \pm 1.3 \mathrm{~mm}, 6.8 \pm 1.1 \mathrm{~mm}$ and $7.5 \pm 1.1 \mathrm{~mm}$ at 3,6 and 12 months postoperatively, respectively ("intraOP" to "3 months": $P=0.0278$, "intraOP" to "12 months": $\mathrm{P}<0.0001)$. Between 12 months and midterm, the APT of the operated knees increased further to $9.0 \pm 1.9 \mathrm{~mm}$ ("12 months" to "lastFU", $P<0.0001)$. A highly significant positive correlation was detected between the APT and KT-1000 measurements at the last follow-up

Table 2 Lysholm rating (in \%)

\begin{tabular}{llll}
\hline Lysholm & Very good & Good & Moderate \\
\hline Frequencies & 35 & 43 & 22 \\
\hline
\end{tabular}


$(P=0.003 ; \mathrm{r}=0.584)$. Details of the APT measurements (in $\mathrm{mm}$ in triplicate) are presented in Fig. 1. At midterm, the calculated side-to-side difference between ACLreconstructed and contralateral uninjured knees in APT measured using the Rolimeter ${ }^{\circledR}$ was $2.1 \pm 1.3 \mathrm{~mm}$; up to $30 \%$ of patients demonstrated a side-to-side difference in APT measurements $\geq 3 \mathrm{~mm}$ using the Rolimeter ${ }^{\circledR}$. All patients showed a firm endpoint during the Lachman test; no patient presented mediolateral joint instability.

The further clinical outcome is presented in the Tables 1,2 and 3 as assessed using the IKDC Grading (Table 1), the Lysholm Rating (Table 2) and the changes in sports activity levels according to Noyes (Table 3). The (median, range) Tegner Activity Level at midterm was 4 (3-9) points. The Lysholm Score at midterm was $89 \pm 9$ points.

Evaluation of conventional radiographs and MR imaging

The measured medial anterior tibial translation in the monopodal stance test was $5.2 \pm 2.4 \mathrm{~mm}$ for affected and $4.0 \pm 1.9 \mathrm{~mm}$ for uninjured contralateral knees. The calculated difference between medial anterior tibial translation in the monopodal stance tests was $1.2 \pm 2.0 \mathrm{~mm}$. Only $15 \%$ of patients demonstrated a side-to-side difference $\geq 3 \mathrm{~mm}$ using the radiographic measurements. The comparison to the amount of ACL-deficient knees (side-toside difference $\geq 3 \mathrm{~mm}$ ) as detected using the APT measurement showed no statistical significant difference (15 vs. $30 \%$, n. s.). In preoperative MRI, all patients showed a total, intraligamentous ACL tear. MRI performed at the last follow-up confirmed the integrity of BPTB grafts used for ACL reconstruction in all cases. The radiological findings according to the IKDC evaluation are presented in Table 4 .

\section{Discussion}

The most important finding of the present study was that restoration of physiological laxity of the transplant in ACL

Table 3 Changes in sports activity levels according to Noyes (in \%)

\begin{tabular}{lllll}
\hline Noyes & Total & $\begin{array}{l}\text { No } \\
\text { symptoms } \\
\text { (Group C) }\end{array}$ & $\begin{array}{l}\text { Moderate } \\
\text { symptoms } \\
\text { (Group D) }\end{array}$ & $\begin{array}{l}\text { No symptoms } \\
\text { in lower } \\
\text { activity levels } \\
\text { (Group E) }\end{array}$ \\
\hline $\begin{array}{c}\text { Returned to same } \\
\text { sports activity } \\
\text { level (Group A) }\end{array}$ & 83 & 68 & 32 & - \\
$\begin{array}{c}\text { Unable to resume } \\
\text { participation in } \\
\text { original sport } \\
\text { (Group B) }\end{array}$ & 17 & - & 50 & 50 \\
\hline
\end{tabular}

Table 4 Radiological findings (in \%)

\begin{tabular}{lllll}
\hline IKDC & $\begin{array}{l}\text { Normal } \\
\text { joint space }\end{array}$ & $\begin{array}{l}>4 \mathrm{~mm} \\
\text { joint space }\end{array}$ & $\begin{array}{l}2-4 \mathrm{~mm} \\
\text { joint space }\end{array}$ & $\begin{array}{l}<2 \mathrm{~mm} \\
\text { joint space }\end{array}$ \\
\hline $\begin{array}{c}\text { Injured } \\
\text { knee }\end{array}$ & 5 & 20 & 75 & - \\
$\begin{array}{c}\text { Uninjured } \\
\text { knee }\end{array}$ & 5 & 15 & 80 & - \\
\hline
\end{tabular}

reconstruction was feasible by means of the Rolimeter and resulted in a satisfying outcome at the midterm. Outcome after ACL reconstruction depends on the initial tension applied to the graft at the time of fixation [31, 33]. Nonphysiologically high initial tension adversely affects ligament matrix remodelling and may lead to reduction in mechanical properties [19] and/or graft failure [14]. Therefore, Tohyama et al. noted that establishing quantitative guidelines for optimal tension of each graft used in ACL reconstruction may be necessary [31]. The present report introduces an intraoperative technique to adjust the APT (as a reference for tension) of the graft in ACLdeficient knees to that of contralateral uninjured knees. Statistical analysis indicated that application of physiological graft tension by APT adjustment using the Rolimeter ${ }^{\circledR}$ was feasible, thus confirming the first part of the hypothesis of the study.

Patients demonstrated an increase in APT by Rolimeter $^{\circledR}$ measurements in reconstructed knees in the early postoperative course after 3 and 12 months and at the midterm, indicative of graft failure in $30 \%$ of patients. However, ACL reconstruction was not considered unsuccessful, as patients did not suffer from subjective instability, and radiographic analysis did not provide evidence for graft rupture at midterm. Thus, the second hypothesis that restoration of physiological laxity prevents from recurrent joint instability has been confirmed partially. Reasons for the preserved joint stability despite the observed APT increase might be as follows: Radiographic assessment of APT in a monopodal stance test indicated side-to-side differences of $\geq 3 \mathrm{~mm}$ in only $15 \%$ of patients. In contrast to the APT measurement assessment in supine position, radiographic assessment of APT under monopodal weight-bearing conditions was less indicative (by trend) for anterior knee instability and supported patients' perceptions in daily activities or sports. The activation of the perigenicular musculature under weightbearing conditions in the radiographic assessment of APT as well as in patients' activities may account for the latter observation. In particular, activation of the hamstrings may underlie this observation: More et al. showed in an in vitro study that increased anterior tibial translation in ACLsectioned knees was reduced if $90-\mathrm{N}$ hamstring load was 
applied [23], indicating that the hamstrings acted as a protagonist to the ACL with potential to compensate for ACL deficiency. This effect was especially observed from $15^{\circ}$ to $45^{\circ}$ knee flexion. Both APT measurements (Rolimeter ${ }^{\circledR}$ and monopodal stance test) were performed within this range at $20^{\circ}$ knee flexion. Furthermore, Woo et al. showed that anterior tibial translation in human cadaveric knees was significantly lower in ACL-reconstructed knees using the patella tendon graft compared to those using a quadrupled semitendinosus-gracilis graft at $15^{\circ}$ and $30^{\circ}$ of flexion in response to a $134-\mathrm{N}$ anterior tibial load [32]. In addition, a meta-analysis revealed that patellar tendon autografts may provide a functionally stable knee more often than hamstring tendon autografts [9, 34]. Increased laxity in ACL-reconstructed knees has been described with the use of hamstrings as a graft source [5, 8]. Moreover, EMG measurements in patients with ACL deficiency demonstrated clearly that hamstrings assume the role of joint stabilizers, leading to the suggestion that hamstring strengthening has significant potential to improve knee stability in patients with ACL deficiency [28].

The third hypothesis has to be rejected as radiological joint deterioration at midterm was not prevented as proposed. However, clinical and radiological outcomes indicated only asymptomatic degenerative changes resulting from the APT increase observed within the first year after reconstruction. The appearance of these degenerative changes from 2 to 5 years after reconstruction using either patellar or hamstring tendons has been observed even previously [12, 13, 20]. APT increase and occurrence of degenerative changes in reconstructed knees at the midterm remain a major concern and may indicate that degenerative arthritis might not be prevented by ACL reconstruction. However, the clinical evaluation revealed a high level of activity and subjective patient satisfaction.

The low number of patients and the very stringent inclusion criteria may be a limitation of the study. However, careful patient selection was used to eliminate factors potentially confounding the outcome assessment. A further limitation may be the loss to follow-up. However, patients satisfied with the clinical results often decline consultation for scientific purposes.

The strengths of the study are that (1) the decisionmaking process, surgical treatment, and APT measurement were performed by only one experienced knee surgeon, (2) clinical and radiological follow-up evaluations were conducted by a blinded and experienced surgeon and radiologist, respectively, and (3) APT measurements were consistent during the follow-up period.

In summary, the present study demonstrates that intraoperative adjustment of the APT in affected knees (to be reconstructed) to the APT of contralateral uninjured knees is feasible. The presented technique provides intraoperative (indirect) control of the applied tension, especially for less experienced surgeons. Anteroposterior stabilization mechanisms of the perigenicular musculature under physiologic dynamic weight-bearing conditions resulted (by trend) in less increase in APT.

\section{Conclusion}

ACL reconstruction using the presented technique was considered successful, as patients did not suffer from subjective instability, radiographic analysis did not provide evidence for graft rupture at midterm. However, APT increase and occurrence of degenerative changes in reconstructed knees at the midterm might not be prevented even by restoration of a physiological APT in ACL reconstruction. In the day by day clinical work, especially the less experienced surgeon might benefit from the presented technique as a direct feedback mechanism for physiological tensioning of the transplant obtaining a satisfying outcome is provided. Moreover, consistent measurements of the APT during follow-up were possible so that the Rolimeter might serve as a convenient and portable arthrometer for follow-up evaluation.

Acknowledgments This work is supported by the "Schweizerische Gesellschaft für Orthopädie und Traumatologie (SGOT)", Tafers, Switzerland. We thank Dr. Daniel Dietrich, Institute of Mathematical Statistics and Actuarial Science, University of Bern, Switzerland, for performing the statistical analysis and his assistance in the interpretation of the results. We also thank Dr. Debra Bickes-Kelleher for linguistic help in the preparation of this manuscript.

\section{References}

1. Abramowitch SD, Papageorgiou CD, Withrow JD, Gilbert TW, Woo SL (2003) The effect of initial graft tension on the biomechanical properties of a healing ACL replacement graft: a study in goats. J Orthop Res 21(4):708-715

2. Aglietti P, Buzzi R, Giron F, Simeone AJ, Zaccherotti G (1997) Arthroscopic-assisted anterior cruciate ligament reconstruction with the central third patellar tendon. A 5-8-year follow-up. Knee Surg Sports Traumatol Arthrosc 5(3):138-144

3. Amis AA, Jakob RP (1998) Anterior cruciate ligament graft positioning, tensioning and twisting. Knee Surg Sports Traumatol Arthrosc 6(Suppl 1):S2-12

4. Beighton P, Horan F (1969) Orthopaedic aspects of the EhlersDanlos syndrome. J Bone Joint Surg Br 51(3):444-453

5. Branch TP, Siebold R, Freedberg HI, Jacobs CA (2011) Doublebundle ACL reconstruction demonstrated superior clinical stability to single-bundle ACL reconstruction: a matched-pairs analysis of instrumented tests of tibial anterior translation and internal rotation laxity. Knee Surg Sports Traumatol Arthrosc 19(3):432-440

6. Dejour H, Bonnin M (1994) Tibial translation after anterior cruciate ligament rupture. Two radiological tests compared. J Bone Joint Surg Br 76(5):745-749

7. Dejour H, Neyret P, Boileau P, Donell ST (1994) Anterior cruciate reconstruction combined with valgus tibial osteotomy. Clin Orthop Relat Res 299:220-228 
8. Feller JA, Webster KE (2003) A randomized comparison of patellar tendon and hamstring tendon anterior cruciate ligament reconstruction. Am J Sports Med 31(4):564-573

9. Freedman KB, D'Amato MJ, Nedeff DD, Kaz A, Bach BR Jr (2003) Arthroscopic anterior cruciate ligament reconstruction: a metaanalysis comparing patellar tendon and hamstring tendon autografts. Am J Sports Med 31(1):2-11

10. Friederich NF, O'Brien WR (1998) Anterior cruciate ligament graft tensioning versus knee stability. Knee Surg Sports Traumatol Arthrosc 6(Suppl 1):S38-S42

11. Ganko A, Engebretsen L, Ozer H (2000) The Rolimeter: a new arthrometer compared with the KT-1000. Knee Surg Sports Traumatol Arthrosc 8(1):36-39

12. Gorschewsky O, Klakow A, Putz A, Mahn H, Neumann W (2007) Clinical comparison of the autologous quadriceps tendon (BQT) and the autologous patella tendon (BPTB) for the reconstruction of the anterior cruciate ligament. Knee Surg Sports Traumatol Arthrosc 15(11):1284-1292

13. Harilainen A, Linko E, Sandelin J (2006) Randomized prospective study of ACL reconstruction with interference screw fixation in patellar tendon autografts versus femoral metal plate suspension and tibial post fixation in hamstring tendon autografts: 5-year clinical and radiological follow-up results. Knee Surg Sports Traumatol Arthrosc 14(6):517-528

14. Hayashi K (1996) Biomechanical studies of the remodeling of knee joint tendons and ligaments. J Biomech 29(6):707-716

15. Hefti F, Muller W, Jakob RP, Staubli HU (1993) Evaluation of knee ligament injuries with the IKDC form. Knee Surg Sports Traumatol Arthrosc 1(3-4):226-234

16. Hui C, Salmon LJ, Kok A, Maeno S, Linklater J, Pinczewski LA (2011) Fifteen-year outcome of endoscopic anterior cruciate ligament reconstruction with patellar tendon autograft for "isolated" anterior cruciate ligament tear. Am J Sports Med 39(1):89-98

17. Irrgang JJ, Ho H, Harner CD, Fu FH (1998) Use of the International Knee Documentation Committee guidelines to assess outcome following anterior cruciate ligament reconstruction. Knee Surg Sports Traumatol Arthrosc 6(2):107-114

18. Jones KG (1963) Reconstruction of the anterior cruciate ligament. A technique using the central one-third of the patellar ligament. J Bone Joint Surg Am 45:925-932

19. Katsuragi R, Yasuda K, Tsujino J, Keira M, Kaneda K (2000) The effect of nonphysiologically high initial tension on the mechanical properties of in situ frozen anterior cruciate ligament in a canine model. Am J Sports Med 28(1):47-56

20. Keays SL, Bullock-Saxton JE, Keays AC, Newcombe PA, Bullock MI (2007) A 6-year follow-up of the effect of graft site on strength, stability, range of motion, function, and joint degeneration after anterior cruciate ligament reconstruction: patellar tendon versus semitendinosus and Gracilis tendon graft. Am J Sports Med 35(5):729-739
21. Lohmander LS, Englund PM, Dahl LL, Roos EM (2007) The long-term consequence of anterior cruciate ligament and meniscus injuries: osteoarthritis. Am J Sports Med 35(10):1756-1769

22. Mae T, Shino K, Nakata K, Toritsuka Y, Otsubo H, Fujie H (2008) Optimization of graft fixation at the time of anterior cruciate ligament reconstruction. Part I: effect of initial tension. Am J Sports Med 36(6):1087-1093

23. More RC, Karras BT, Neiman R, Fritschy D, Woo SL, Daniel DM (1993) Hamstrings - an anterior cruciate ligament protagonist. An in vitro study. Am J Sports Med 21(2):231-237

24. Noyes FR, Barber SD, Mooar LA (1989) A rationale for assessing sports activity levels and limitations in knee disorders. Clin Orthop Relat Res 246:238-249

25. Pernin J, Verdonk P, Si Selmi TA, Massin P, Neyret P (2010) Long-term follow-up of 24.5 years after intra-articular anterior cruciate ligament reconstruction with lateral extra-articular augmentation. Am J Sports Med 38(6):1094-1102

26. Sanders TG, Miller MD (2005) A systematic approach to magnetic resonance imaging interpretation of sports medicine injuries of the knee. Am J Sports Med 33(1):131-148

27. Schuster AJ, McNicholas MJ, Wachtl SW, McGurty DW, Jakob RP (2004) A new mechanical testing device for measuring anteroposterior knee laxity. Am J Sports Med 32(7):1731-1735

28. Solomonow M, Baratta R, Zhou BH, Shoji H, Bose W, Beck C, D'Ambrosia R (1987) The synergistic action of the anterior cruciate ligament and thigh muscles in maintaining joint stability. Am J Sports Med 15(3):207-213

29. Staubli HU, Rauschning W (1994) Tibial attachment area of the anterior cruciate ligament in the extended knee position. Anatomy and cryosections in vitro complemented by magnetic resonance arthrography in vivo. Knee Surg Sports Traumatol Arthrosc 2(3):138-146

30. Tegner Y, Lysholm J (1985) Rating systems in the evaluation of knee ligament injuries. Clin Orthop Relat Res 198:43-49

31. Tohyama H, Yasuda K (1998) Significance of graft tension in anterior cruciate ligament reconstruction. Basic background and clinical outcome. Knee Surg Sports Traumatol Arthrosc 6(Suppl 1):S30-S37

32. Woo SL, Kanamori A, Zeminski J, Yagi M, Papageorgiou C, Fu FH (2002) The effectiveness of reconstruction of the anterior cruciate ligament with hamstrings and patellar tendon. A cadaveric study comparing anterior tibial and rotational loads. J Bone Joint Surg Am 84(A 6):907-914

33. Yasuda K, Tsujino J, Tanabe Y, Kaneda K (1997) Effects of initial graft tension on clinical outcome after anterior cruciate ligament reconstruction. Autogenous doubled hamstring tendons connected in series with polyester tapes. Am J Sports Med 25(1):99-106

34. Yunes M, Richmond JC, Engels EA, Pinczewski LA (2001) Patellar versus hamstring tendons in anterior cruciate ligament reconstruction: a meta-analysis. Arthrosc 17(3):248-257 